\title{
Versatile synthesis of probes for high-throughput enzyme activity screening
}

\author{
Tristan de Rond • Pamela Peralta-Yahya • \\ Xiaoliang Cheng • Trent R. Northen • Jay D. Keasling
}

Received: 17 January 2013 /Revised: 24 February 2013 /Accepted: 28 February 2013 / Published online: 10 April 2013

(C) The Author(s) 2013. This article is published with open access at Springerlink.com

\begin{abstract}
Mass spectrometry based technologies are promising as generalizable high-throughput assays for enzymatic activity. In one such technology, a specialized enzyme substrate probe is presented to a biological mixture potentially exhibiting enzymatic activity, followed by an in situ enrichment step using fluorous interactions and nanostructureinitiator mass spectrometry. This technology, known as Nimzyme, shows great potential but is limited by the need to synthesize custom substrate analogs. We describe a
\end{abstract}

Electronic supplementary material The online version of this article (doi:10.1007/s00216-013-6888-z) contains supplementary material, which is available to authorized users.

T. de Rond

Department of Chemistry, University of California,

Berkeley, CA 94720, USA

T. de Rond $\cdot$ P. Peralta-Yahya $\cdot$ J. D. Keasling

California Institute for Quantitative Biosciences,

University of California, Berkeley, CA 94720, USA

P. Peralta-Yahya $\cdot$ X. Cheng $\cdot$ T. R. Northen $\cdot$ J. D. Keasling

Joint BioEnergy Institute, 5885 Hollis Street,

Emeryville, CA 94608, USA

X. Cheng · T. R. Northen · J. D. Keasling

Physical Biosciences Division, Lawrence Berkeley National

Laboratory, Berkeley, CA 94720, USA

X. Cheng $\cdot$ T. R. Northen

Life Sciences Division, Lawrence Berkeley National Laboratory,

Berkeley, CA 94720, USA

\section{J. D. Keasling $(\bowtie)$}

Chemical and Biomolecular Engineering and Bioengineering

Departments, University of California, Berkeley, CA 94720, USA

e-mail: keasling@berkeley.edu

\section{Present Address:}

P. Peralta-Yahya

Department of Chemistry and Biochemistry,

Georgia Institute of Technology, 901 Atlantic Drive,

Atlanta, GA 30332, USA synthetic route that simplifies the production of these probes by fashioning their perfluorinated invariant portion as an alkylating agent. This way, a wide variety of compounds can be effectively transformed into enzyme activity probes. As a proof of principle, a chloramphenicol analog synthesized according to this methodology was used to detect chloramphenicol acetyltransferase activity in cell lysate. This verifies the validity of the synthetic strategy employed and constitutes the first reported application of Nimzyme to a non-carbohydrate-active enzyme. The simplified synthetic approach presented here may help advance the application of mass spectrometry to high-throughput enzyme activity determination.

Keywords Enzyme assays · High-throughput · Nimzyme · Nanostructure-initiator mass spectrometry ·

Chloramphenicol acetyltransferase

\section{Introduction}

Given the biochemical and biotechnological significance of enzymes, high-throughput assays for the detection of enzymatic activity are in high demand. High-throughput enzyme activity assays are critical in the drug and biomarker discovery processes and can be a valuable tool for functional gene annotation. They figure prominently in directed evolution experiments, where libraries of enzyme variants are screened for improved substrate specificity, thermal stability, or other beneficial properties.

High-throughput enzyme activity assays can help alleviate experimental bottlenecks, but few generally applicable technologies are currently available. Some enzymatic reactions can be coupled to a spectroscopic readout by employing chromogenic substrates or by indirectly measuring product formation via a coupled assay or biosensor. Such assays are fast, but applicable to only the narrow range 
of biochemical transformations for which methods have been developed. On the other hand, mass spectrometrybased assays are more universal, but require lengthy chromatographic separations to avoid spectral complexity and ionization suppression, drastically reducing experimental throughput (Fig. 1a).

Nimzyme is a nanostructure-initiator mass spectrometry (NIMS)-based analytical technique that can detect enzymatic activity in complex mixtures such as crude cell lysate [1-3]. It circumvents time-intensive chromatographic separations by means of an in situ fluorous affinity purification (Fig. 1b). This, in combination with acoustic sample deposition [4], shows potential as a high-throughput enzyme activity assay.

Currently, two hurdles limit the applications of Nimzyme. First, the technique requires the chemical synthesis of a specialized analog for each substrate of interest [5]. Second, all reported applications of Nimzyme thus far have been on enzymes acting on carbohydrates [1-4]. Hence, the generalizability of the technique has yet to be demonstrated.
Here, we report a synthetic route towards Nimzyme probes in which the substrate moiety is introduced towards the end of the synthesis. Accordingly, the invariant portion of these compounds needs to be synthesized only once. By fashioning this fragment as an alkylating agent, a variety of synthetically and biologically significant molecules can be readily transformed into Nimzyme probes. We also show that a chloramphenicol analog synthesized according to this methodology is effective in detecting chloramphenicol acetyltransferase (CAT) activity in crude cell lysate.

\section{Experimental}

General synthetic remarks and materials

Moisture-free conditions were employed only where indicated. Reagents and solvents were purchased from TCI America, ChemPep, Alfa Aesar, BDH, and Sigma-Aldrich,

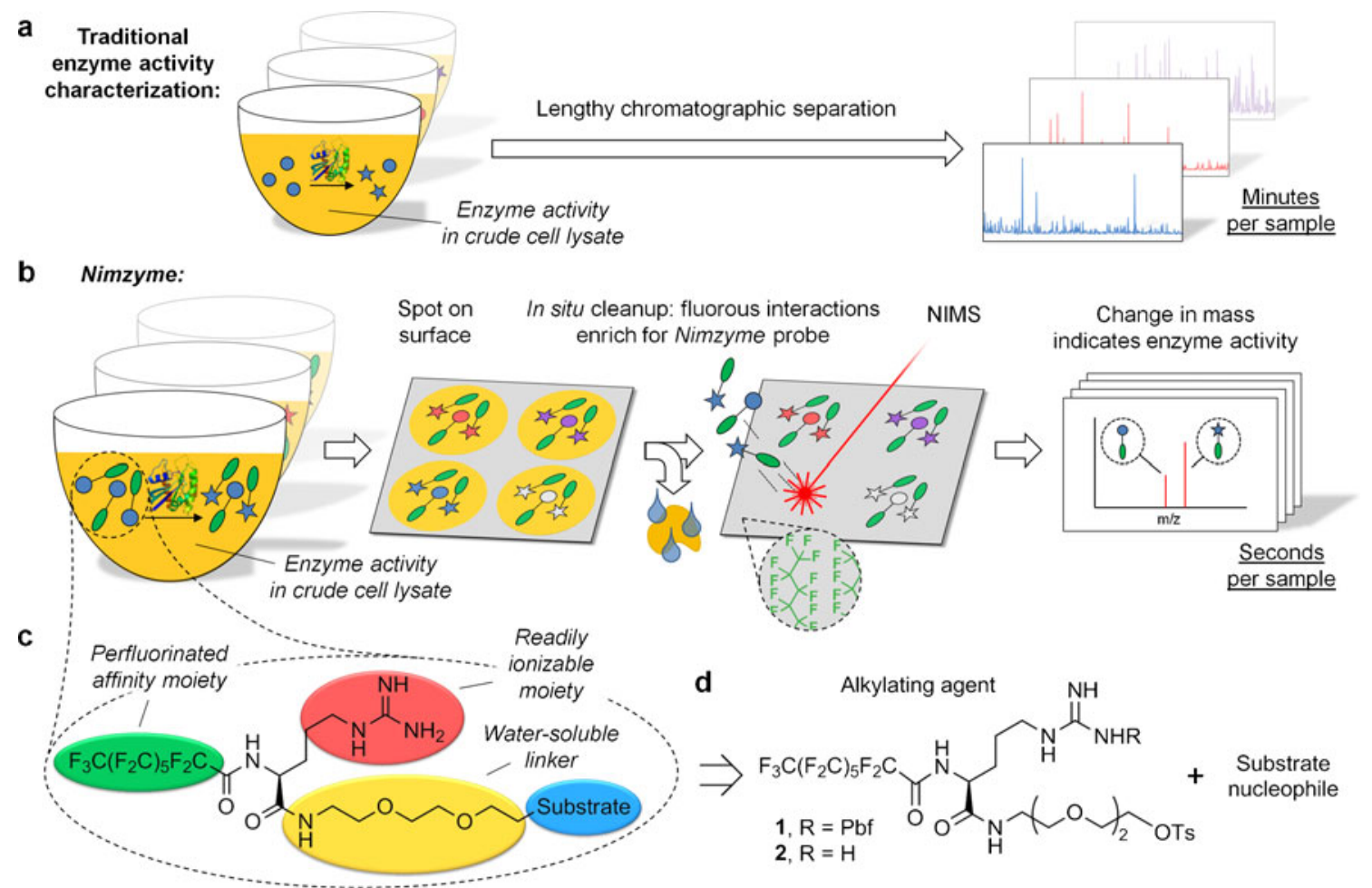

Fig. 1 Overview of the Nimzyme technology. a Performing protein purification on a large number of samples is usually prohibitive. Hence, high-throughput activity assays are typically performed in whole cells or in cell lysate, which requires separation by chromatography before mass spectrometric analysis. b Workflow of the highthroughput Nimzyme enzyme activity assay as described in ref. [2]. Enzyme substrate analogs harboring perfluoroalkyl moieties are incubated in a complex mixture exhibiting enzymatic activity (e.g., crude cell lysate). The mixture is quenched and spotted (e.g., using acoustic deposition) on a NIMS chip coated with a perfluorinated initiator substance. The surface is subjected to aqueous washes, allowing cell debris to be rinsed away while fluorous interactions with the chip surface retain the analytes. Subsequent NIMS, performed on a MALDI-TOF mass spectrometer, generates mass spectra where the ratio of substrate to product mass speaks is a measure of enzyme activity. c Generalized anatomy of a Nimzyme substrate. Substrate analogs that can be employed in Nimzyme require a perfluorinated moiety to allow for in situ cleanup and an ionizable group to improve NIMS sensitivity. d The retrosynthetic strategy employed in this manuscript. The invariable part of these substrates is synthesized once, in the form of tosylate alkylating agents $\mathbf{1}$ and $\mathbf{2}$. These can react with a wide variety of nucleophiles to provide Nimzyme-amenable substrate analogs. Key: $T s=p$-toluenesulfonyl; $P b f=2,2,4,6,7$ pentamethyldihydrobenzofuran-5-sulfonyl 
anhydrous whenever possible, and were used as received unless otherwise indicated. FluoroFlash fluorous solidphase extraction cartridges were purchased from Fluorous Technologies Incorporated.

\section{Design of alkylating agents}

A generalized structure of Nimzyme-amenable substrate analogs as prepared in this manuscript is shown in Fig. 1c. A perfluoroalkanoyl moiety imparts the required fluorophilic character to allow for on-chip purification, and an arginine moiety ensures high ionization efficiency in NIMS [1]. Avoiding reliance on the substrate moiety to impart ionizability also allows for direct comparison of NIMS peak heights to determine relative abundance of chemical species. A tri(ethylene glycol) linker was installed to improve enzyme-substrate accessibility and enhance the probe's solubility in water.

Nucleophilic substitution chemistry was chosen to couple substrates of interest to the probe's invariant portion because many biologically relevant substrates possess nucleophilic functional groups. Hence, we incorporated a $p$-toluenesulfonate ester (tosylate) leaving group into the invariant portion of the Nimzyme probe, resulting in alkylating agents 1 and 2 (Fig. 1d).

Synthesis of alkylating agents

A synthetic route to 1 and 2 was devised, which starts from readily available starting materials. A synthetic scheme, detailed synthetic protocols, and spectral data of all intermediates are reported in the Electronic Supplementary Material (Scheme S1 and S2 and Figs. S1-S11). Briefly, 2-(2-(2-aminoethoxy)ethoxy)ethanol-obtained by means of a Gabriel synthesis from 2-(2-(2-chloroethoxy)ethoxy) ethanol-was coupled to Fmoc- $\mathrm{Arg}(\mathrm{Pbf})-\mathrm{OH}$ using conventional solution-phase peptide synthesis methodology. The Fmoc group was removed with diethylamine and the resulting amine was acylated with perfluorooctanoyl chloride under Schotten-Baumann conditions. The resulting alcohol was tosylated to afford 1 in $26 \%$ yield with respect to Fmoc$\mathrm{Arg}(\mathrm{Pbf})-\mathrm{OH}$ (four steps). Lastly, the Pbf protecting group was removed with 90:10 TFA/MeOH to afford 2.

\section{Alkylation reactions}

The alkylation and deprotection reactions were monitored by NIMS of $0.1 \mu \mathrm{L}$ of the reaction mixture dissolved in $10 \mu \mathrm{L}$ methanol. Fluorous solid-phase extraction (FSPE) was performed on FluoroFlash $2 \mathrm{~g}$ cartridges as follows: The cartridges were preconditioned with $1 \mathrm{~mL}$ $\mathrm{N}, \mathrm{N}$-dimethylformamide (DMF) and $7 \mathrm{~mL}$ 80:20 $\mathrm{MeOH} / \mathrm{H}_{2} \mathrm{O}$, loaded with the crude alkylation reaction mixture, washed with $7 \mathrm{~mL}$ 80:20 MeOH/ $\mathrm{H}_{2} \mathrm{O}$, and eluted with
$10 \mathrm{~mL} \mathrm{MeOH}$. Each F-SPE cartridge was used only once. Possible product isomerism was ruled out by LC/MS (see ESM). Because the quantities prepared here are too small to be accurately weighed, their yields were determined relative to a known concentration of Nz-OMe (12 in the ESM) by mixing them in a $1: 1$ ratio and determining the ratio of NIMS peak intensities. 12 was chosen as an internal standard because its mass does not overlap with any of the reagents, products, or possible side products and can reasonably be assumed to ionize similarly to 3 through 11 . For amine nucleophiles (products 5, 6, and 11), using the hydrochloride form led to the formation of what was presumed to be "Nz(Pbf)-Cl" (NIMS calc'd for $[\mathrm{M}+\mathrm{H}]^{+}$972.2; found 972.1; characteristic 3:1 M+H:M+H+2 ratio). Hence, we either purchased or generated the free base form of these substrates. Representative reactions using alkylating agents 1 and 2 are described in detail below. All others are described in the ESM; ' $N z$ ' $=2-\left(2-\left(N^{\alpha}\right.\right.$-perfluorooctanoylargininamidoethoxy) ethoxy)ethyl (structural formula shown in Fig. 2a)

\section{9-Ibuprofen $\mathrm{Nz}$ ester}

Into a borosilicate test tube with stir bar, $5.7 \mathrm{mg}$ ibuprofen sodium salt ( $25 \mu \mathrm{mol}, 50 \mathrm{eq}), 10 \mu \mathrm{L} 50 \mathrm{mM} \mathrm{Nz-OTs}(2)$ in DMF, and $490 \mu \mathrm{L}$ DMF were added. The mixture was stirred at $70{ }^{\circ} \mathrm{C}$ for $3 \mathrm{~h}$. After cooling to room temperature (RT), $5 \mathrm{~mL} 1 \mathrm{M}$ aqueous $\mathrm{NaOH}$ was added, the resulting solution was extracted with $3 \times 5 \mathrm{~mL}$ chloroform, and each of the extracts was in turn washed with another $5 \mathrm{~mL} 1 \mathrm{M}$ aqueous $\mathrm{NaOH}$. A few crystals of $\mathrm{NH}_{3} \mathrm{Cl}$ were added to the chloroform extracts, which were filtered and evaporated in vacuo to yield a colorless residue which was taken up in $\mathrm{MeOH} 78$ \% yield.

\section{1-(1R,2R)-N-Nz-1-(4-nitrophenyl)propane-1,3-diol}

Into a borosilicate test tube with stir bar, $50 \mu \mathrm{L} 100 \mathrm{mM}$ $\mathrm{Nz}(\mathrm{Pbf})-\mathrm{OTs}(\mathbf{1})$ in DMF, $200 \mu \mathrm{L}$ more DMF, and $53.1 \mathrm{mg}$ (250 $\mu \mathrm{mol}, 50$ eq) $(1 R, 2 R)$-2-amino-1-(4-nitrophenyl)-1,3propanediol were added. The mixture was stirred at $110{ }^{\circ} \mathrm{C}$ for $2 \mathrm{~h}$, cooled to RT, and F-SPE purified. The eluent was evaporated in vacuo, redissolved in $2 \mathrm{~mL}$ 90:10 TFA/MeOH, and left to stir at RT for $12 \mathrm{~h}$. The TFA/MeOH was evaporated under a gentle stream of nitrogen, leaving a white residue which was taken up in $\mathrm{MeOH} 45 \%$ yield.

NIMS surface fabrication

The production of NIMS chips has been described elsewhere [6]. Briefly, a silicon wafer is cleaned thoroughly with methanol, followed by anodic etching with $25 \%$ hydrofluoric acid $(w / v)$ in ethanol in a custom-made Teflon etching chamber using a current of $2.4 \mathrm{~A}$ for $15 \mathrm{~min}$. Next, the chips are coated by 


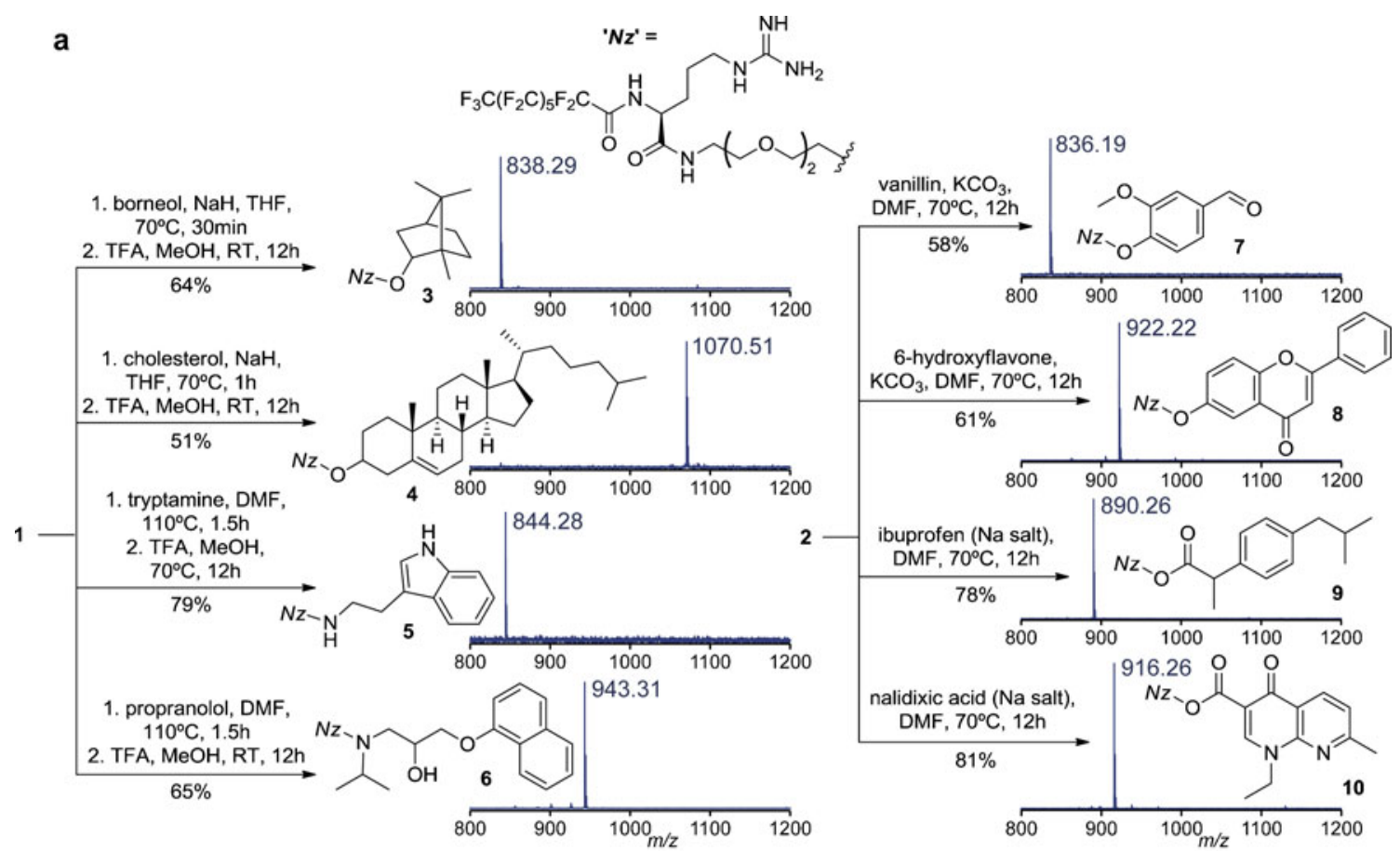

b<smiles>CC(=O)OC[C@H](NC(=O)C(Cl)Cl)C(O)c1ccc([N+](=O)[O-])cc1</smiles>

C

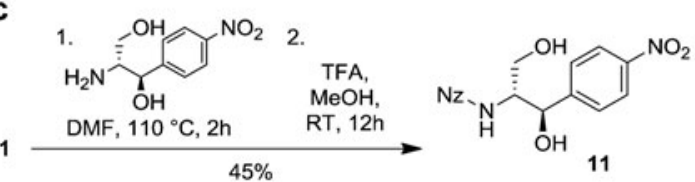

d
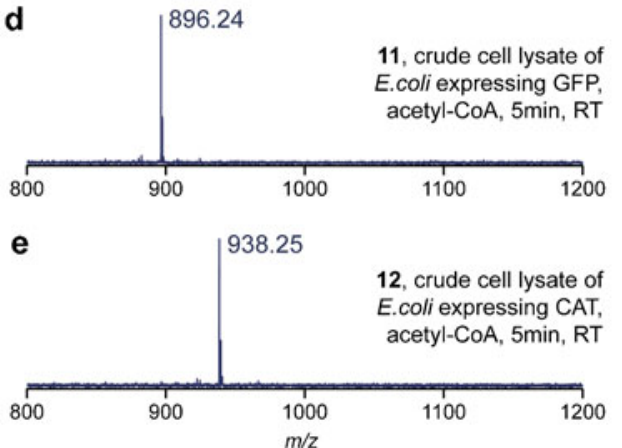

Fig. 2 Convenient synthesis of Nimzyme probes and their suitability for the detection of CAT activity in cell lysate. a Synthesis of Nimzyme substrates using tosylates $\mathbf{1}$ and $\mathbf{2}$ as alkylating agents, and NIMS spectra of the products purified only by F-SPE (for 1), or liquid-liquid extraction (for 2). Yields reported are combined yields for alkylation, purification, and (for 1) deprotection. b CAT catalyzes the $O^{3}$-acetylation of chloramphenicol. c Chloramphenicol analog $\mathbf{1 1}$ was synthesized through the alkylation of $(1 R, 2 R)$-2-amino-1-(4-nitrophenyl)-1,3- propanediol with $\mathbf{1}$ according to the methodology shown. d Exposure of $\mathbf{1 1}$ to control lysate followed by the Nimzyme workflow shown in Fig. 1b shows a clean mass peak corresponding to $\mathbf{1 1}$ (expected $\mathrm{M}+\mathrm{H}, m / z=896.26$ ). e Exposure of $\mathbf{1 1}$ to lysate of $E$. coli having expressed CAT, followed by Nimzyme, shows a mass shift of exactly one acetyl unit relative to $\mathbf{1 1}$ (expected $\mathrm{M}+\mathrm{H}, \mathrm{m} / \mathrm{z}=$ 938.26). Key: $T H F=$ tetrahydrofuran; $T F A=$ trifluoroacetic acid; $D M F=N, N^{\prime}$-dimethylformamide

sodium phosphate ( $\mathrm{pH} 7.5)$, and sonicated for $30 \mathrm{~s}$. AcetylCoA was added to a final concentration of $2 \mathrm{mM}$ and 11 to a final concentration of $0.5 \mathrm{mM}$. The mixture was mixed and incubated at room temperature for $5 \mathrm{~min}$ and subsequently quenched with an equal volume of methanol. A $0.3-\mu \mathrm{L}$ droplet of the mixture was spotted onto a NIMS chip and the excess liquid removed $5 \mathrm{~s}$ later by touching it with a Kimwipe (Kimberly-Clark). The spotted area was washed with $2 \times 1 \mu \mathrm{L}$ deionized water. The NIMS chip was taped to a modified standard matrix-assisted laser desorption/ionization (MALDI) plate, which was then loaded into an Applied Biosystems 4800 MALDI time of flight (TOF)/TOF mass spectrometer. Agilent ESI-L Low Concentration Tuning Mix 
was spotted nearby on the NIMS chip to allow for mass calibration of the instrument. Spectra were acquired in manual mode and positive polarity.

\section{Results}

\section{Alkylation reactions}

We investigated the capability of 1 and 2 to react with a number of biologically relevant nucleophiles (Fig. 2a). It was found that 1 was able to alkylate alcohols by means of a Williamson ether synthesis, forming Nimzyme probes 3 and 4. Amines were likewise readily alkylated by 1 to form 5 and 6 , provided the free base form was used. We used excess nucleophile to drive the reactions and to avoid overalkylation of amines. The alkylated intermediates could be recovered using F-SPE, circumventing cumbersome chromatographic purifications. Subsequent deprotection of the Pbf group affords the desired Nimzyme probes in good yield. Alkylating agent 2 was found to react readily with carboxylate salts and phenolates to directly form Nimzyme probes 7-10. We were unable to find conditions under which these could be purified by F-SPE, and hence, we chose to remove excess nucleophile using basic aqueous washes.

\section{Detection of chloramphenicol acetyltransferase activity}

To verify that the substrate analogs synthesized according to the described methodology could be used to detect enzymatic activity in a Nimzyme assay, we synthesized chloramphenicol analog 11 as a probe for CAT activity (Fig. 2b, c). CAT catalyzes the transfer of an acetyl group from acetyl-CoA to $O^{3}$ of chloramphenicol [7]. When 11 is exposed to a control $E$. coli cell lysate, Nimzyme reveals a clean mass peak corresponding to this substrate (Fig. 2d). When instead 11 is exposed to lysate from $E$. coli overexpressing CAT, the peak shifts by 42 mass units, as expected from monoacetylation (Fig. 2e). This result verifies the validity of the synthetic strategy employed and furthermore comprises the first reported application of Nimzyme to a non-carbohydrate-active enzyme.

\section{Discussion}

In summary, we present the synthesis of a pair of alkylating agents and conditions under which they can be used to transform a wide range of structures into Nimzyme probes. The alkylation reactions and subsequent purifications are undemanding, such that an inexperienced chemist can perform them with minimal effort. One probe synthesized according to this methodology, a chloramphenicol analog, could serve to detect CAT activity in cell lysate, verifying the synthetic approach and broadening the scope of the Nimzyme technology. The other synthesized probes were not tested in enzymatic reactions in this work.

The strategy described here allows for the construction of libraries of Nimzyme substrates without the need to repeatedly re-synthesize the invariable portion of the probes. By streamlining access to compatible enzyme substrates, and by showing that Nimzyme can be applied to non-carbohydrateactive enzymes, we hope to have cleared two of the barriers to the widespread adoption of this technology for highthroughput enzyme characterization. Certain drawbacks remain to be solved, such as the possibility that the modifications required of substrates to be compatible with Nimzyme affect enzyme-substrate binding interactions. While this has been shown not to be the case for $\beta$-glycosidases [2], enzymes with sufficiently buried active sites may or may not accept the probes presented here as substrates. Work to overcome these shortcomings is currently ongoing.

Acknowledgments This publication was made possible by grant number 1RC1GM090980-01 from the National Institutes of Health. Its contents are solely the responsibility of the authors and do not necessarily represent the official views of the NIH. This work was part of the DOE Joint BioEnergy Institute (http://www.jbei.org), supported by the U.S. Department of Energy, Office of Science, Office of Biological and Environmental Research, through contract DE-AC02-05CH11231 between Lawrence Berkeley National Laboratory and the U.S. Department of Energy.

Open Access This article is distributed under the terms of the Creative Commons Attribution License which permits any use, distribution, and reproduction in any medium, provided the original author(s) and the source are credited.

\section{References}

1. Northen TR, Lee JC, Hoang L, Raymond J, Hwang DR, Yannone SM, Wong CH, Siuzdak G (2008) A nanostructure-initiator mass spectrometrybased enzyme activity assay. Proc Natl Acad Sci USA 105:3678-3683

2. Reindl W, Deng K, Gladden JM, Cheng G, Wong A, Singer SW, Singh S, Lee JC, Yao CH, Hazen TC, Singh AK, Simmons BA, Adams PD, Northen TR (2011) Colloid-based multiplexed screening for plant biomass-degrading glycoside hydrolase activities in microbial communities. Energy Environ Sci 4:2884-2893

3. Deng K, George KW, Reindl W, Keasling JD, Adams PD, Lee TS, Singh AK, Northen TR (2012) Encoding substrates with mass tags to resolve stereospecific reactions using Nimzyme. Rapid Commun Mass Spectrom 26:611-615

4. Greving M, Cheng X, Reindl W, Bowen B, Deng K, Louie K, Nyman M, Cohen J, Singh A, Simmons B, Adams P, Siuzdak G, Northen T (2012) Acoustic deposition with NIMS as a high-throughput enzyme activity assay. Anal Bioanal Chem 403:707-711

5. Fernandez-Arrojo L, Guazzaroni ME, Lopez-Cortes N, Beloqui A, Ferrer M (2010) Metagenomic era for biocatalyst identification. Curr Opin Biotechnol 21:725-733

6. Woo HK, Northen TR, Yanes O, Siuzdak G (2008) Nanostructure-initiator mass spectrometry: a protocol for preparing and applying NIMS surfaces for high-sensitivity mass analysis. Nat Protoc 3:1341-1349

7. Shaw WV (1984) Bacterial resistance to chloramphenicol. Br Med Bull 40:36-41 